\title{
Comparative effectiveness of adjuvant treatments for resected gastric cancer: a network meta-analysis
}

\author{
Zhaolun Cai $^{1}$ - Yiqiong Yin ${ }^{1} \cdot$ Yuan Yin ${ }^{1}$. Chaoyong Shen ${ }^{1}$. Jian Wang ${ }^{1} \cdot$ Xiaonan Yin $^{1} \cdot$ Zhixin Chen $^{1} \cdot$ Ye Zhou $^{1}$. \\ Bo Zhang ${ }^{1}$
}

Received: 2 February 2018 / Accepted: 27 April 2018 / Published online: 4 May 2018

(c) The International Gastric Cancer Association and The Japanese Gastric Cancer Association 2018

\begin{abstract}
Background Different adjuvant treatments are available for patients with gastric cancer, but conventional meta-analyses performing direct comparisons between two alternative treatments did not have enough power to compare all the adjuvant treatments. Thus, we did a network meta-analysis summarizing the direct and indirect comparisons to identify the optimum treatment.

Methods We systematically searched for RCTs of adjuvant treatments for gastric cancer comparing two or more of the following treatments: surgery alone, radiotherapy with fluoropyrimidine, S-1-based regimens, and XELOX. The treatments offering available indirect evidence to investigate the comparative effectiveness of adjuvant treatments mentioned above were also included. Then we performed a Bayesian network meta-analysis to summarize the direct and indirect comparisons. We estimated hazard ratios with 95\% credible intervals (CrI) for OS and DFS.

Results 11 eligible RCTs (5620 patients) were included in the network meta-analysis. Radiotherapy with fluorouracil (5-FU/ RT), S-1-based regimens, and XELOX significantly improved OS as compared with surgery alone [(HR $=0.75$ with $95 \%$ CrI: 0.63-0.89), (HR $=0.63$ with 95\% CrI: $0.52-0.76)$, and ( $\mathrm{HR}=0.66$ with $95 \% \mathrm{CrI}$ : $0.51-0.85)$, respectively]. No treatment was clearly superior to others; however, S-1-based regimes and XELOX showed a statistically non-significant trend to better survival as compared with 5-FU/RT.

Conclusions S-1-based chemotherapy and XELOX are likely to be the most effective adjuvant treatments for patients with resected gastric cancer. 5-FU alone provided little survival benefits as compared with surgery alone. Further clinical trials may be required to investigate $\mathrm{S}$-1-based and XELOX-based adjuvant treatment strategies.
\end{abstract}

Keywords Gastric cancer · Adjuvant · Chemotherapy $\cdot$ Chemoradiotherapy, network meta-analysis

Zhaolun Cai and Yiqiong Yin contributed equally to the research.

Electronic supplementary material The online version of this article (https://doi.org/10.1007/s10120-018-0831-0) contains supplementary material, which is available to authorized users.

Bo Zhang

hxwcwk@126.com

Zhaolun Cai

caizhaolun@foxmail.com

Yiqiong Yin

yiqiong489@163.com

Yuan Yin

Yinyuan10@gmail.com

Chaoyong Shen

scyshenchaoyong@163.com

Jian Wang

wj66220@126.com
Xiaonan Yin

yxnyinxiaonan@163.com

Zhixin Chen

chenzhixin@medmail.com.cn

Ye Zhou

zhouye79@yahoo.com

1 Department of Gastrointestinal Surgery, West China Hospital, Sichuan University, Chengdu 610041, Sichuan, China 


\section{Introduction}

Gastric cancer is the fifth most common cancer worldwide and the third most common cause of death from cancer, accounting for $6.8 \%$ of the total cases and $8.8 \%$ of total deaths with about 723,000 deaths in 2012 [1]. Surgery is the only curative treatment in patients with localized gastric cancer [2], the overall survival (OS) remains poor for locoregional as well as distant recurrence after curative resection [3]. Thus, various adjuvant treatments including chemotherapy and chemoradiotherapy have been investigated to improve the surgical outcomes and prevent recurrence of the disease during the past decades, which was confirmed by several meta-analyses [4-6]. Efficacy has also been established for several adjuvant treatments in randomized controlled trials (RCTs) [7-12]. The National Comprehensive Cancer Network (NCCN) guideline for the treatment of gastric cancer advised capecitabine and oxaliplatin (XELOX) as adjuvant chemotherapy regimens based on the results of the CLASSIC trial [7, 13] and radiotherapy with fluoropyrimidine as adjuvant chemoradiotherapy based on the INT-0116 trial [11]. On the other hand, S-1-based regimes and XELOX are recommended in Japanese gastric cancer treatment guidelines for the results of the ACTS-GC, J-CLASSIC, and SOX-adjuvant trials [8, 14-16]. Adjuvant chemotherapy including XELOX and S-1 and adjuvant chemoradiotherapy are both recommended in European Society for Medical Oncology (ESMO) guidelines [2].

However, most of those postoperative adjuvant treatments have never been compared with each other for the lack of head-to-head trials and the limitation of traditional meta-analysis methods which could only perform direct pairwise comparisons. Thus, the most effective adjuvant treatment for resected gastric cancer is still unknown. D2 gastrectomy has been the standard of care for advanced gastric cancer in East Asia [14, 17] and recommended in western guidelines [2, 13] and the optimum treatment for patients undergoing D2 lymph node dissection also remains controversial. On the other hand, how to select different adjuvant treatment for the stage III gastric cancer has been the focus of public attention and identifying the more effective postoperative treatments for stage III disease is urgently needed.

We used a Bayesian network meta-analysis to investigate the questions. In the Bayesian hierarchical model, comparisons of two or more treatments are available by using indirect comparisons when there are no head-tohead, comparative studies, we can overcome the shortage of direct comparison trials and combine direct and indirect comparisons to compare several inventions at the same time [18-21]. The aim of our network meta-analysis was to investigate and summarize the direct and indirect comparisons to derive the comparative effect of all included adjuvant treatments for resected gastric cancer.

\section{Method}

\section{Search strategy}

Two investigators performed a systematic literature search in PubMed, EMBASE (Ovid), Cochrane Library (Ovid) (last updated on May 30, 2017) without language restriction, using combinations of the following terms: "stomach neoplasms", "gastric cancer", "stomach cancer", "esophagogastric junction", "gastroesophageal junction", "adjuvant”, "Postoperative", "chemotherapy, adjuvant", "Randomized Controlled Trial", "Controlled Clinical Trial" in accordance with the Cochrane Handbook for Systematic Reviews of Interventions [22].

The reference list was also checked for relevant studies, and all studies were carefully evaluated to identify duplicate data.

\section{Study selection}

The following criteria were used for the study selection: (1) participants $(\mathrm{P})$ : patients were eligible if they had histologically proven gastric or gastroesophageal junction adenocarcinoma with no evidence of distant metastasis. (2) Interventions (I) and comparisons (C): we only included the RCTs of adjuvant treatments for gastric cancer comparing 2 or more of the following treatments: surgery alone, radiotherapy with fluoropyrimidine, S-1-based regimes, XELOX. The RCTs of adjuvant treatments offering available indirect evidence other than treatments mentioned above were also enrolled in the meta-analysis. Patients enrolled in the studies had no previous chemotherapy or radiotherapy. (3) Outcomes: OS, (the time between randomization and all-cause death) or disease-free survival (DFS), time between randomization and the first event of all-cause death, relapse of stomach cancer, or occurrence of a second cancer); (4) study design (S): published randomized controlled trials; (5) provided enough information to estimate hazard ratio (HR) and $95 \%$ confidence intervals (CIs) of OS or DFS.

Conference abstracts, letters, case reports, reviews, studies without randomization for treatment allocation or studies without usable data were excluded. Studies concerning intraperitoneal chemotherapy or immunotherapy were also excluded.

For the subgroup analysis for D2 lymph node dissection, only studies with extractable data of patients with D2 lymph node dissection were included. Studies with extractable data of patients with stage III gastric cancer were included in the 
subgroup analysis of stage III gastric cancer, staging classification was according to the American Joint Committee on Cancer (AJCC) cancer staging manual (sixth edition) [23].

\section{Assessment of risk of bias and data collection}

Qualitative assessment and data extraction were finished by two investigators independently. Disagreements were resolved in discussion with a third investigator. The two researchers used the same standardized collection form to independently extract information from each enrolled study. We also extracted the HR and 95\% CI to assess OS and DFS. Data concerning study quality, population characteristics and year of publication as well as interventions and outcomes were extracted.

The quality and the risk of bias of randomized controlled trials was assessed by Cochrane Collaboration's tool [24].

\section{Statistical analysis}

The meta-analysis was performed according to PRISMA checklist [25]. The primary outcome of our network metaanalysis was OS, and the secondary outcomes were DFS. HRs which take the number and timing of events into consideration with its $95 \%$ CI were used to assess time-to-event outcomes, we obtained the data directly from the studies or used Kaplan-Meier survival curves to estimate the HRs of survival, which is reported by Tierney et al $[22,26]$. 95\% credible intervals $(\mathrm{Crl})$ of $\mathrm{HR}$ for the estimates the network meta-analyses. An HR below 1 indicated a better prognosis with the experimental intervention.

The network meta-analyses using the Bayesian methods [18] was performed in Stata 12 (Stata Corp, College Station, TX, USA), JAGS and R (version x64 3.3.3) with the gemtc package (version: 0.8-2) and rjags package (version: 4-6) with a fixed-effect model, as most of the head-to-head comparisons only included one trial providing direct evidence.

The inconsistency of our results was also confirmed by the node-splitting method and its Bayesian P value [27], comparing the direct and the indirect estimates for each comparison. We estimated the potential ranking probability of treatments by calculating the surface under the cumulative ranking curve (SUCRA) for each intervention [28]. The SUCRA index ranges between 0 (or 0\%) and 1 (or 100\%), where the treatments with higher SUCRA values are considered to have better efficacy.

\section{Result}

\section{Study selection and characteristics}

We systematically searched PubMed, EMBASE, the Cochrane Library for RCTs. A total of 3712 articles were considered to be potentially relevant and 11 studies [7-11, 29-34] meeting the inclusion criteria were included in this meta-analysis. Literature screening process is shown in Fig. 1.

The characteristics of included studies are summarized in Table 1. Seven treatments were compared, and the network plots of all the comparisons analyzed are shown in Fig. 2; each of nodes included different interventions with specific plan of treatments: fluorouracil with or without leucovorin (5-FU); radiotherapy with fluorouracil (5-FU/RT); capecitabine, oxaliplatin (XELOX); cisplatin, epirubicin/epidoxorubicin, leucovorin, and fluorouracil (PELF); S-1-based regimes; UFT-based regimes. The size of the nodes and the thickness of the edges are weighted according to the number of studies evaluating each treatment and direct comparison, respectively. In total, our analysis included 5620 patients: 1769 treated with surgery alone; 283, 5-FU; 499, 5-FU/RT; 520, XELOX; 220, PELF; 1234, S-1-based regimes; 1095, UFT-based regimes.

\section{Network meta-analysis}

\section{Overall survival}

All the 11 trials contributed to our network meta-analysis of OS, comparing the 7 treatments. HRs were explicitly reported in 9 trials and also could be estimated in 2 trials [31, 34]. In Fig. 3A, we summarize the comparisons analyzed by the Bayesian network meta-analysis. Four treatments which were shown to have a significantly improved prognosis compared with surgery only were 5-FU/RT $[\mathrm{HR}=0.75$ with $95 \%$ CrI: $(0.63,0.89)], S-1$-based regimens [HR $=0.63$ with $95 \%$ CrI: $(0.52,0.76)]$, UFT-based regimens $[\mathrm{HR}=0.75$ with $95 \%$ CrI: $(0.61,0.92)]$ and XELOX $[\mathrm{HR}=0.66$ with $95 \% \mathrm{CrI}$ : $(0.51,0.85)]$, and the rest of the adjuvant treatment strategies, were not associated with an improved OS when compared with surgery alone. 5-FU/RT, S-1-based regimens, and XELOX also yielded longer OS than 5-FU. No statistical difference was found between 5-FU/RT, S-1-based regimens and XELOX in OS.

The SUCRA values of 92 and $81 \%$ for S-1-based regimens and XELOX suggested that these were the two treatments with the highest chance of improving OS in resected gastric cancer. 5-FU and surgery only had the least chance of improving OS (Table 2).

\section{Disease-free survival}

Nine trials [7-11, 29, 30, 32, 34] contributed to our network meta-analysis of DFS, comparing the 7 treatments. HRs were explicitly reported in 7 trials and could be estimated in 2 trials [30, 34]. In Fig. 3A, we summarize the comparisons analyzed by the Bayesian network meta-analysis. 
Fig. 1 Study flow diagram

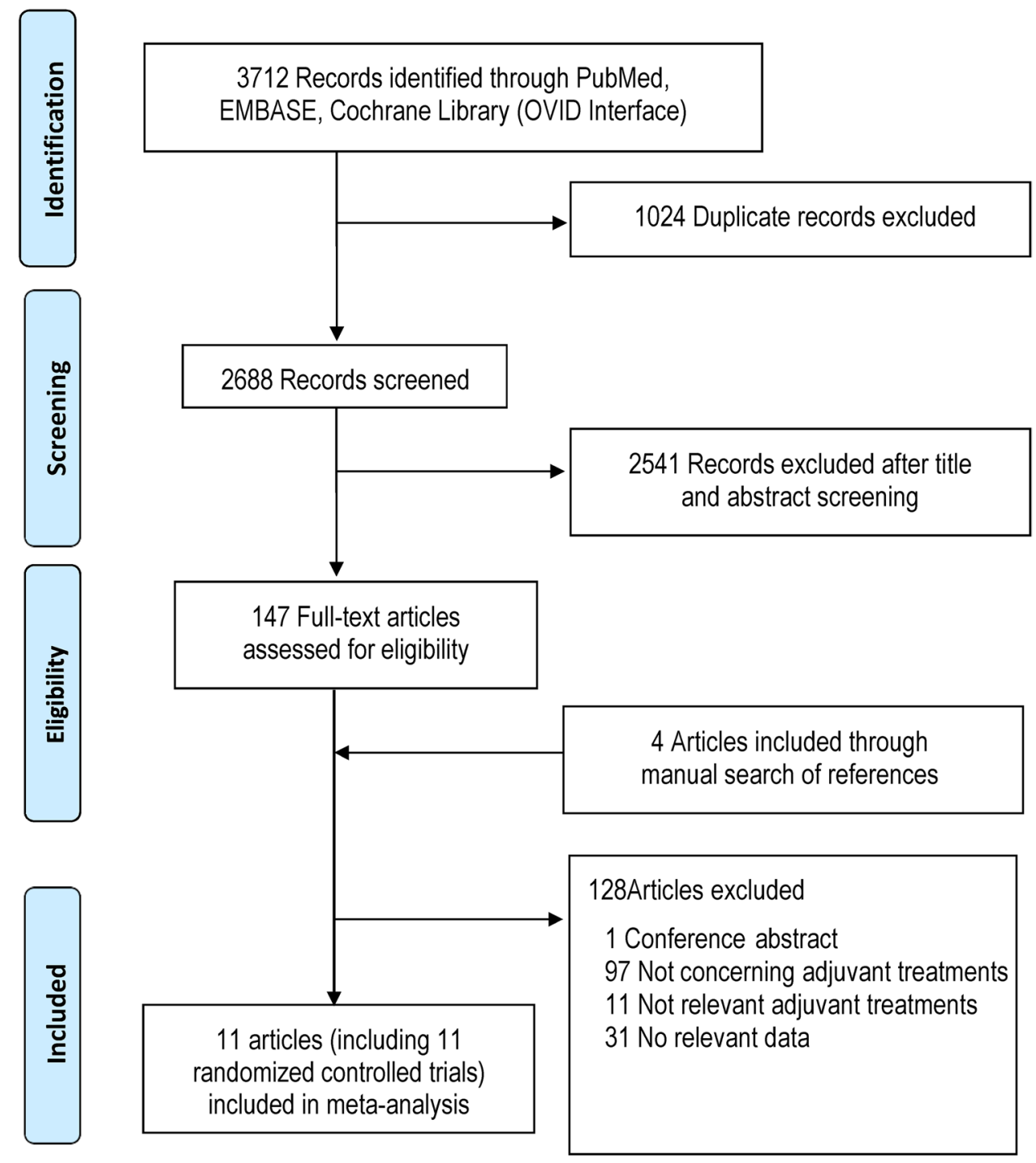

Four treatments which were shown to have a significantly improved prognosis compared with surgery only were 5-FU/ RT $[H R=0.66$ with $95 \%$ CrI: $(0.55,0.80)]$, S-1-based regimens $[\mathrm{HR}=0.60$ with $95 \% \mathrm{CrI}:(0.50,0.72)]$, UFT-based regimens $[\mathrm{HR}=0.71$ with $95 \% \mathrm{CrI}:(0.57,0.88)]$ and XELOX $[\mathrm{HR}=0.58$ with $95 \% \mathrm{CrI}:(0.47,0.72)]$. The rest of the treatments showed no statistical difference when compared with surgery alone. 5-FU/RT, S-1-based regimens, and XELOX yielded longer DFS than 5-FU and PELF. On comparative effectiveness network meta-analysis of 5-FU/RT, S-1-based regimens, and XELOX, no treatment was clearly superior to others.

The SUCRA values of 89 and $86 \%$ for XELOX and S-1based regimens, respectively, suggesting that these were the two treatments with the highest chance of improving DFS in resected gastric cancer, whereas 5-FU and surgery alone was least likely to be the best treatment strategy (SUCRA: $21 \%$ and SUCRA: $8 \%$, respectively) (Table 2).

\section{D2 lymph node dissection}

Five trials [7, 8, 10, 11, 29] reporting available data were included for meta-analysis of adjuvant treatments for resected gastric cancer with D2 lymph node dissection, comparing 5 treatments. The INT-0116 trial included several subgroups, the subset with D2 group was used for the analysis of OS for patients with D2 lymph node dissection [11].

We summarized the results of our subgroup analysis for OS and DFS in Fig. 3B. Compared with surgery alone, S-1-based regimens, UFT-based regimens, and XELOX improved both OS and DFS. 5-FU/RT, however, demonstrated not enough statistical evidence available to support survival benefits for the patients with D2 level of resection when compared with surgery alone in terms of OS $[\mathrm{HR}=0.93$ with $95 \% \mathrm{CrI}:(0.41,2.1)]$.

There was no statistically significant difference between S-1-based regimens and XELOX. The SUCRA 
Table 1 Study and patient population characteristics of included studies

\begin{tabular}{|c|c|c|c|c|c|c|c|}
\hline Author (trial) & Year & $\begin{array}{l}\text { Sample size } \\
\text { (intervention/ } \\
\text { control) }\end{array}$ & Intervention & Control & Lymphadenectomy & Median, age & $\begin{array}{l}\text { Median } \\
\text { follow-up } \\
\text { (months) }\end{array}$ \\
\hline \multicolumn{8}{|c|}{ Adjuvant CT plus surgery vs surgery } \\
\hline ACTS-GC & 2011 & $515 / 519$ & S-1 & Surgery alone & D2 & NR & 36 \\
\hline CLASSIC & 2014 & $520 / 515$ & $\begin{array}{l}\text { Capecitabine, oxali- } \\
\text { platin }\end{array}$ & Surgery alone & D2 & NR & 34 \\
\hline Di Costanzo, F & 2008 & $130 / 128$ & $\begin{array}{l}\text { Cisplatin, epirubicin/ } \\
\text { epidoxorubicin, leuco- } \\
\text { vorin, fluorouracil }\end{array}$ & Surgery alone & $\mathrm{D} 1 / \mathrm{D} 2 / \mathrm{D} 3 / \mathrm{D} 4$ & 59 & 67.2 \\
\hline Nakajima, T & 2007 & $93 / 95$ & UFT & Surgery alone & $\mathrm{D} 1 / \mathrm{D} 1+$ & 63 & 45.6 \\
\hline Nakajima, T & 1999 & $288 / 285$ & $\begin{array}{l}\text { Mitomycin, fluorouracil, } \\
\text { UFT }\end{array}$ & Surgery alone & NR & NR & 80.4 \\
\hline \multicolumn{8}{|c|}{ Adjuvant CT plus surgery vs adjuvant CT plus surgery } \\
\hline Cascinu, $\mathrm{S}$ & 2007 & $58 / 59$ & $\begin{array}{l}\text { Cisplatin, epirubicin/ } \\
\text { epidoxorubicin, leuco- } \\
\text { vorin, fluorouracil }\end{array}$ & Leucovorin, fluorouracil & D1/D2 & $58 / 59$ & 54 \\
\hline Lee, J. J & 2004 & $32 / 29$ & $\begin{array}{l}\text { Cisplatin, epirubicin/ } \\
\text { epidoxorubicin, leuco- } \\
\text { vorin, fluorouracil }\end{array}$ & Fluorouracil & D2 & $52.5 / 52$ & 42 \\
\hline Tsuburaya, A & 2014 & $719 / 714$ & $\begin{array}{l}\text { S-1 } \\
\text { S-1, paclitaxel }\end{array}$ & $\begin{array}{l}\text { UFT } \\
\text { UFT, paclitaxel }\end{array}$ & D2 & NR & 62.5 \\
\hline \multicolumn{8}{|c|}{ Adjuvant CRT plus surgery vs adjuvant CT plus surgery } \\
\hline Kwon, H. C & 2010 & $31 / 30$ & $\begin{array}{l}\text { Leucovorin, fluoroura- } \\
\text { cil, Radiotherapy }\end{array}$ & Leucovorin, fluorouracil & D2 & 52.5 & 77.2 \\
\hline Zhu, W. G & 2012 & $186 / 165$ & $\begin{array}{l}\text { Leucovorin, fluoroura- } \\
\text { cil, Radiotherapy }\end{array}$ & Leucovorin, fluorouracil & D2 & 57 & 42.5 \\
\hline \multicolumn{8}{|c|}{ Adjuvant CRT plus surgery vs surgery } \\
\hline INT-0116 & 2012 & $282 / 227$ & $\begin{array}{l}\text { Leucovorin, fluoroura- } \\
\text { cil, Radiotherapy }\end{array}$ & Surgery alone & $\mathrm{D} 0 / \mathrm{D} 1 / \mathrm{D} 2$ & NR & 123.6 \\
\hline
\end{tabular}

$C T$ chemotherapy, $C R T$ chemoradiotherapy, $N A$ not available, $N R$ not reported

values for XELOX and S-1-based regimens suggested that these were the two treatments with the highest chance of improving OS and DFS in resected gastric cancer (Table 2).

\section{Stage III gastric cancer}

Two trials $[7,8]$ reporting available data were included for subgroup analysis of adjuvant treatments for resected stage III gastric cancer, comparing surgery alone, S-1 and XELOX. Results of subgroup analysis of stage III disease are shown in Table 3. Either S-1 or XELOX had a significantly improved OS $[\mathrm{HR}=0.73$ with $95 \% \mathrm{CrI}$ : $(0.54$, $0.98)$ and $\mathrm{HR}=0.72$ with $95 \%$ CrI: $(0.53,0.98)$, respectively] and DFS [HR $=0.66$ with $95 \%$ CrI: $(0.50,0.87)$ and $\mathrm{HR}=0.58$ with $95 \% \mathrm{CrI}:(0.44,0.76)$, respectively] compared with surgery alone. There was no statistically significant difference between XELOX and S-1 for stage III disease in terms of OS and DFS.

\section{Quality of evidence}

The bias assessment for eligible RCTs included in the network meta-analysis is shown in Online Resource 1 according to the Cochrane risk-of-bias tool, showing no severe risk of bias.

The result of the comparison-adjusted funnel plots for both OS and DFS in Fig. 4 did not show the evidence of apparent asymmetry, suggesting the absence of publication bias [35]. Tests for funnel plot asymmetry of the two subgroups were not conducted for the reason that there were fewer than 10 studies in both subgroups [36]. We also used the node-splitting analysis of outcomes with $P$ value to confirm the consistency in any closed loops of the two outcomes; the results are shown in Online Resource 2. A P value $<0.05$ indicates a significant inconsistency. According to the results, no consistency in any closed loop was detected with relevant $\mathrm{P}$ value lager than 0.05 by nodesplitting method. 


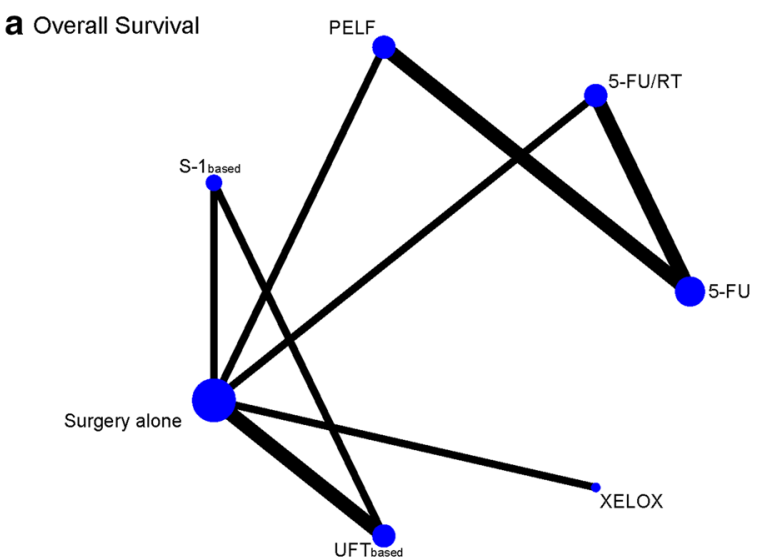

C Subgroup analysis for D2 lymph node dissection

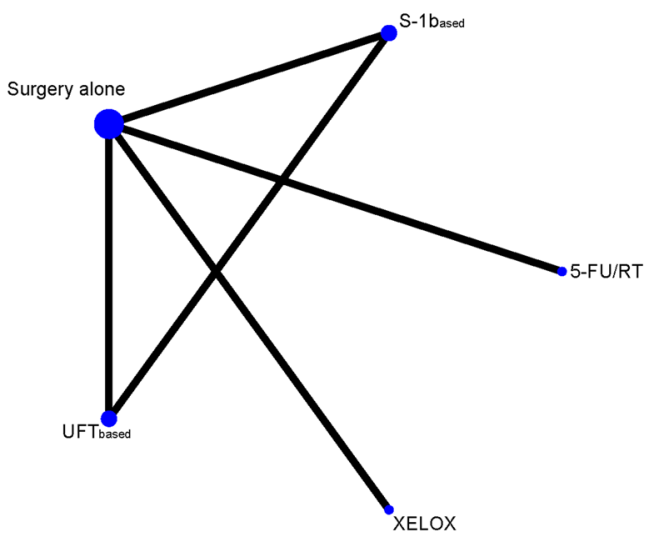

Fig. 2 Network of the comparisons for the Bayesian network metaanalysis. a Overall survival for the general population of patients with gastric cancer; b disease-free survival for the general population of

The absence of inconsistency between the direct and indirect comparison in all networks which was confirmed by both the node-splitting method coupled with the results of the bias assessment and the comparison-adjusted funnel plots allowed assessment of the network meta-analysis.

\section{Discussion}

To give valuable suggestions for choice of treatments through comparing their efficacy, we conducted the network meta-analysis to compare adjuvant treatments for gastric cancer in terms of OS and DFS. Our network meta-analysis is the first study to estimate the HRs for OS and DFS of pairwise comparisons of potential adjuvant treatments for gastric cancer and to provide the relative treatments ranking which serves as supportive information to explore the optimum treatment.

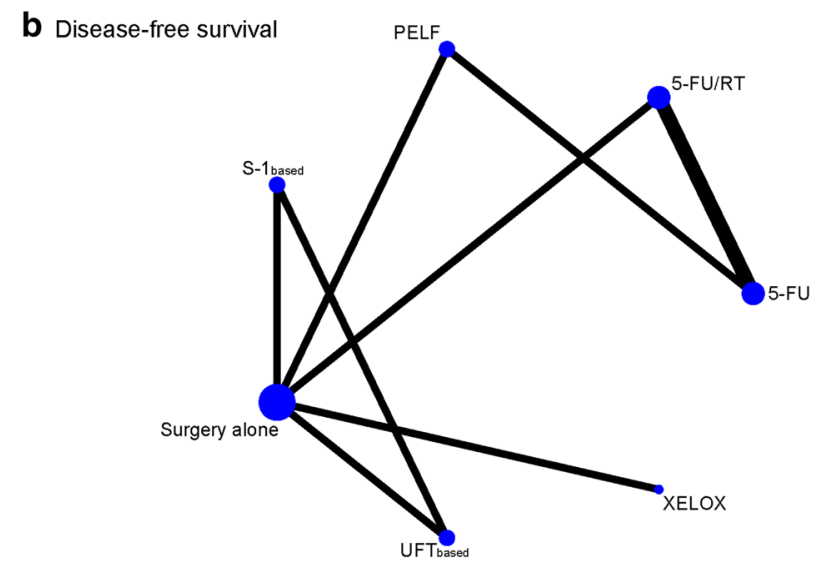

d Subgroup analysis for Stage III gastric cancer

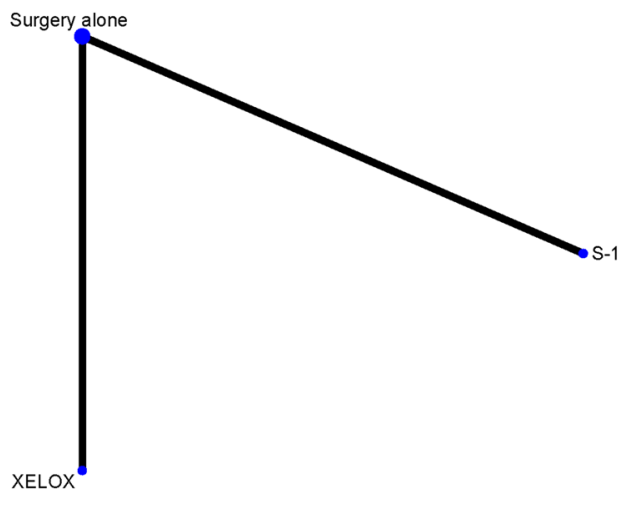

patients with gastric cancer; c subgroup analysis for D2 lymph node dissection; d subgroup analysis for Stage III gastric cancer

In the network meta-analysis, we combined direct and indirect evidence from 11 RCTs (11 for OS, 9 for DFS; 5 for subgroup analysis of the D2 lymph node dissection; 2 for subgroup analysis of stage III gastric cancer) and reported 3851 participants with resected gastric cancer followed by adjuvant treatments.

In the analysis of OS, our results suggested that adjuvant treatments with 5-FU/RT, S-1-based regimens, and XELOX provided an advantage over surgery alone, and the rest of the adjuvant therapies were not associated with an improved OS when compared with surgery alone. In accordance with the findings of the previous study [29], our study demonstrated that UFT-based regimes were inferior to S-1-based regimes. No treatment was clearly superior to others between 5-FU/RT, S-1-based regimens; however, adjuvant S-1based regimes and adjuvant XELOX showed a statistically non-significant trend to better survival as compared with 5-FU/RT. Taking the results of SUCRA into consideration, we suggested that S-1-based regimens and XELOX were 
Fig. 3 Comparative effectiveness of adjuvant treatments for gastric cancer in network metaanalysis. a The general population of patients with resected gastric cancer; b patients with D2 lymphadenectomy. Hazard ratio (95\% credible interval) for comparisons are in cells in common between column-defining and row-defining treatment. Bold cells are significant. For overall survival, hazard ratio $<1$ favors row-defining treatment. For disease-free survival, hazard ratio $<1$ favors columndefining treatment a

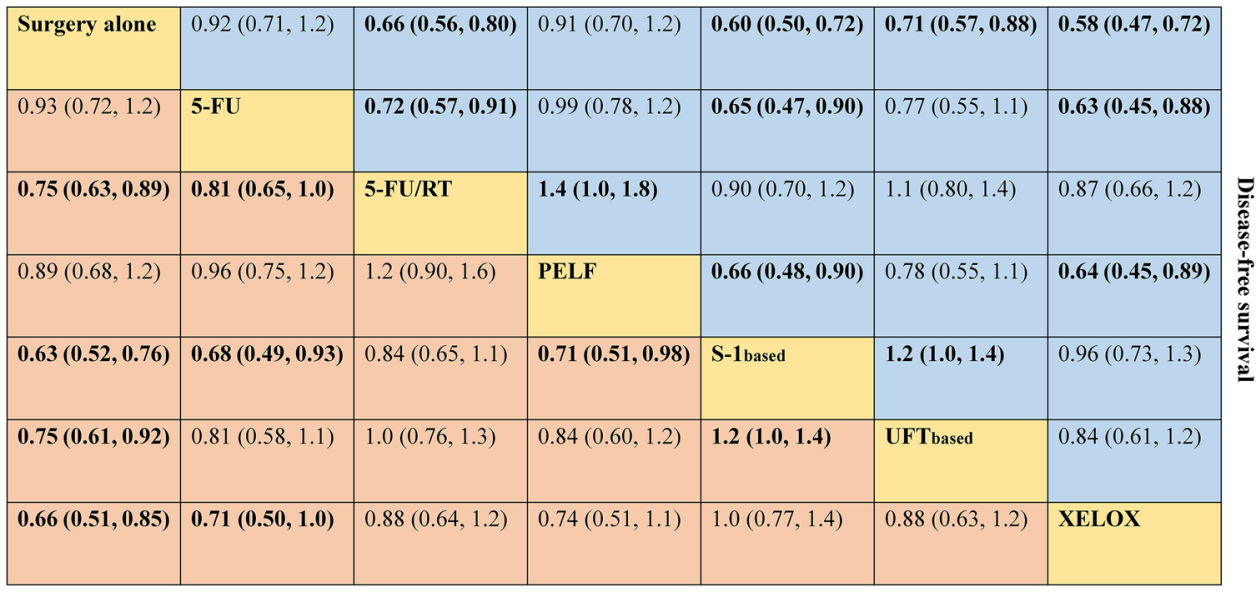

Overall Survival

b

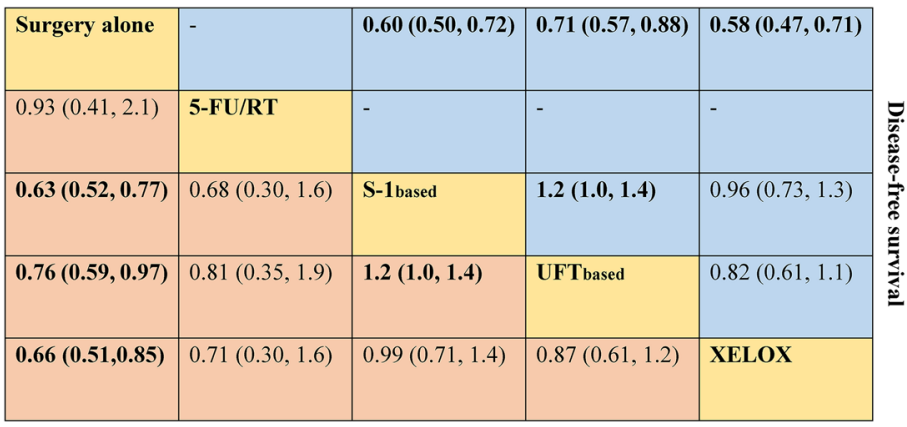

Overall Survival

\begin{tabular}{llllllll}
\hline Treatments & PELF & S-1 based & Surgery alone & UFT based & XELOX & 5-FU & 5-FU/RT \\
\hline OS & 0.30 & 0.92 & 0.08 & 0.57 & 0.81 & 0.21 & 0.60 \\
DFS & 0.23 & 0.86 & 0.086 & 0.55 & 0.89 & 0.20 & 0.67 \\
OS for D2 & - & 0.85 & 0.11 & 0.48 & 0.73 & - & 0.32 \\
DFS for D2 & - & 0.80 & 0 & - & 0.83 & 0.33 & - \\
\hline
\end{tabular}

Table 2 Surface under the cumulative ranking curve (SUCRA) results of OS and DFS 
Table 3 Subgroup analysis: OS and DFS in patients with Stage III gastric cancer

\begin{tabular}{llllll}
\hline Comparisons & \multicolumn{2}{l}{ Overall survival } & & \multicolumn{2}{l}{$\begin{array}{l}\text { Disease-free } \\
\text { survival }\end{array}$} \\
\cline { 6 - 7 } \cline { 5 - 6 } \cline { 5 - 6 } & HR & $95 \%$ CrI & & HR & $95 \%$ CrI \\
\hline Stage III & & & & & \\
S-1 vs surgery alone & $\mathbf{0 . 7 3}$ & $\mathbf{0 . 5 4 - 0 . 9 8}$ & & $\mathbf{0 . 6 6}$ & $\mathbf{0 . 5 0 - 0 . 8 7}$ \\
XELOX vs surgery alone & $\mathbf{0 . 7 2}$ & $\mathbf{0 . 5 3 - 0 . 9 8}$ & & $\mathbf{0 . 5 8}$ & $\mathbf{0 . 4 4 - 0 . 7 6}$ \\
XELOX vs S-1 & 1.0 & $0.66-1.5$ & & 0.88 & $0.60-1.3$ \\
Stage IIIA & & & & & \\
S-1 vs surgery alone & $\mathbf{0 . 6 6}$ & $\mathbf{0 . 4 6 - 0 . 9 6}$ & $\mathbf{0 . 6 3}$ & $\mathbf{0 . 4 5 - 0 . 8 9}$ \\
XELOX vs surgery alone & 0.75 & $0.52-1.1$ & & $\mathbf{0 . 6 1}$ & $\mathbf{0 . 4 4 - 0 . 8 5}$ \\
XELOX vs S-1 & 1.1 & $0.67-1.9$ & & 0.97 & $0.60-1.6$ \\
Stage IIIB & & & & & \\
S-1 vs surgery alone & 0.86 & $0.51-1.4$ & & 0.71 & $0.45-1.1$ \\
XELOX vs surgery alone & 0.67 & $0.39-1.2$ & & $\mathbf{0 . 5 2}$ & $\mathbf{0 . 3 3 - 0 . 8 2}$ \\
XELOX vs S-1 & 0.78 & $0.37-1.7$ & & 0.73 & $0.38-1.4$ \\
\hline
\end{tabular}

For the subgroup analysis of stage III disease, there was no statistically significant difference between XELOX and S-1 in OS and DFS, suggesting the similar efficacy of the two treatments. How to select different adjuvant treatment for the stage III gastric cancer has been the focus of public attention in recent years and numerous trials have been conducted to explore the effectiveness of adjuvant chemotherapies such as S-1 plus oxaliplatin, S-1 plus docetaxel and S-1 plus cisplatin for stage III disease [16, 38-40], reporting contrasting and sometimes equivocal results. The different trends in the adjuvant treatments for stage III gastric cancer warrant further evaluation in RCTs.

Nonetheless, some limitations in the present work merit further discussion. First, several RCTs were conducted

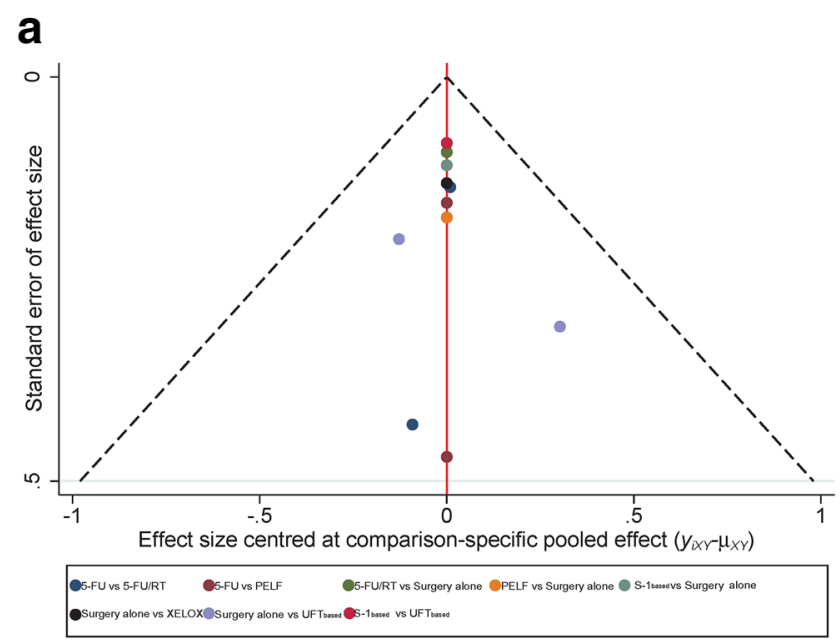

over 2 decades and changes in the medical environment could particularly affect the control arms, which would influence the validity of the results. Second, our study was based on the meta-analysis of aggregate data from longitudinal clinical trials rather than individual patient data (IPD). Thus it was difficult for us to extract, calculate and compare survival data in the subgroups stratified by supposed predictors such as time span and stage classification. Staging modalities involved in the study varying across trials may potentially affect the results. However, only two trials reporting available data were included for subgroup analysis of stage III gastric cancer, the stage classification was according to the sixth edition of the AJCC cancer staging manual [23], which resulted in slightly different population characteristics between this study and the current research [41]. Third, two trials [12, 42] investigating adjuvant platinum/docetaxel chemotherapy with or without radiation therapy and adjuvant capecitabine/cisplatin with or without radiation therapy were excluded for the shortage of trials which could connect the network nodes.

Despite these limitations, there are several strengths of our study. The absence of statistically significant inconsistency and publication bias in network meta-analysis confirmed the accuracy of our results and to the best of our knowledge, and this is the first attempt to systematically and quantitatively review the literature in this field. Moreover, inclusion criteria for enrolled trials were very similar, producing homogeneous populations and study characteristics for our study with the patients with resected gastric cancer.

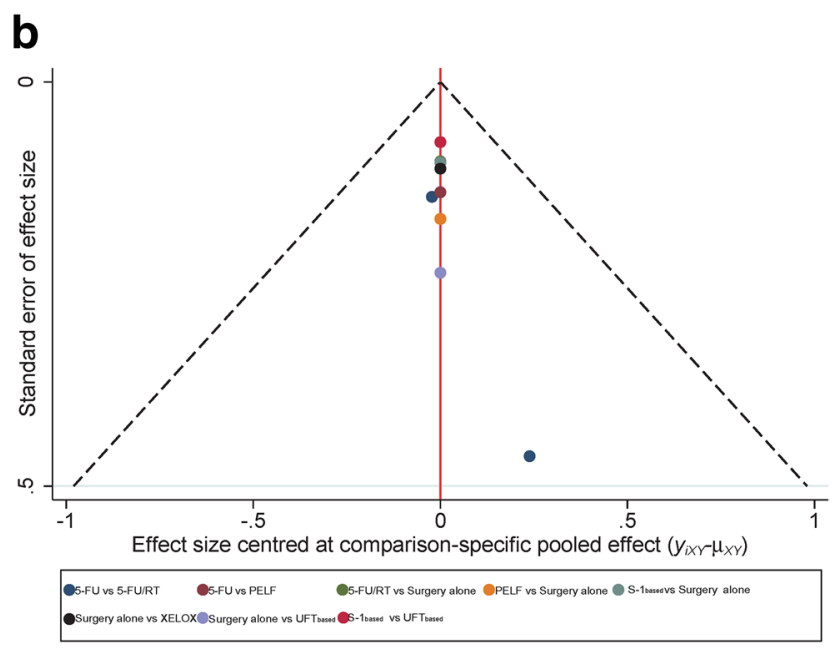

Fig. 4 Comparison-adjusted funnel plots of publication bias test for overall survival and disease-free survival. a Overall survival; b disease-free survival 


\section{Conclusion}

Our network meta-analysis provided the first comparisons between adjuvant treatments for patients with resected gastric cancer. S-1-based chemotherapy and XELOX are likely to be the most effective adjuvant treatments for patients with resected gastric cancer. 5-FU alone provided little survival benefits as compared with surgery alone. Further clinical trials may be required to investigate S-1-based and XELOXbased adjuvant treatment strategies.

Author contributions Bo Zhang, Zhaolun Cai, Yiqiong Yin designed the study. Jian Wang and Xiaonan Yin screened studies and extracted data. Disagreements were resolved by discussion with Yuan Yin. Zhaolun Cai did the statistical analyses and prepared figures. Bo Zhang, Zhixin Chen, Ye Zhou, Yuan Yin, Chaoyong Shen, Zhaolun Cai reviewed the results, interpreted data, and wrote the manuscript. All authors saw and approved the final version of the paper.

Funding There was no sponsor for this study.

\section{Compliance with Ethical Standards}

Conflict of interest The authors declare no conflict of interest.

Ethical statement This article does not contain any studies with human or animal subjects performed by any of the authors.

\section{References}

1. Ferlay J, Soerjomataram I, Dikshit R, Eser S, Mathers C, Rebelo $\mathrm{M}$, et al. Cancer incidence and mortality worldwide: Sources, methods and major patterns in GLOBOCAN 2012. Int J Cancer. 2015;136(5).

2. Smyth EC, Verheij M, Allum W, Cunningham D, Cervantes A, Arnold D, et al. Gastric cancer: ESMO Clinical Practice Guidelines for diagnosis, treatment and follow-up. Ann Oncol. 2016;27(suppl 5):v38-v49. https://doi.org/10.1093/annonc/ mdw350 (Epub 2016/09/25)

3. Dangelica MI, Gonen M, Brennan MF, Turnbull AD, Bains MS, Karpeh MS. Patterns of initial recurrence in completely resected gastric adenocarcinoma. Ann Surg. 2004;240(5):808-16.

4. Paoletti X, Oba K, Burzykowski T, Michiels S, Ohashi Y, Pignon JP, et al. Benefit of adjuvant chemotherapy for resectable gastric cancer: a meta-analysis. Jama. 2010;303(17):1729-37. https://doi. org/10.1001/jama.2010.534. (Epub 2010/05/06)

5. Diaz-Nieto R, Orti-Rodriguez R, Winslet M. Post-surgical chemotherapy versus surgery alone for resectable gastric cancer. The Cochrane database of systematic reviews. 2013;9:CD008415. https://doi.org/10.1002/14651858.CD008415.pub2 (Epub 2013/09/04)

6. Zhou ML, Kang M, Li GC, Guo XM, Zhang Z. Postoperative chemoradiotherapy versus chemotherapy for R0 resected gastric cancer with D2 lymph node dissection: an up-to-date meta-analysis. World J Surg Oncol. 2016;14(1):209. https://doi.org/10.1186/ s12957-016-0957-7 (Epub 2016/08/10)

7. Noh SH, Park SR, Yang HK, Chung HC, Chung IJ, Kim $\mathrm{SW}$, et al. Adjuvant capecitabine plus oxaliplatin for gastric cancer after D2 gastrectomy (CLASSIC]: 5-year follow-up of an open-label, randomised phase 3 trial. Lancet Oncol. 2014;15(12):1389-96. https://doi.org/10.1016/s1470 (Epub 2014/12/03)

8. Sasako M, Sakuramoto S, Katai H, Kinoshita T, Furukawa $\mathrm{H}$, Yamaguchi T, et al. Five-year outcomes of a randomized phase III trial comparing adjuvant chemotherapy with S-1 versus surgery alone in stage II or III gastric cancer. J Clin Oncol. 2011;29(33):4387-93. https://doi.org/10.1200/jco.2011.36.5908 (Epub 2011/10/20)

9. Di Costanzo F, Gasperoni S, Manzione L, Bisagni G, Labianca $\mathrm{R}$, Bravi S, et al. Adjuvant chemotherapy in completely resected gastric cancer: a randomized phase III trial conducted by GOIRC. J Natl Cancer Inst. 2008;100(6):388-98. https://doi.org/10.1093/ jnci/djn054 (Epub 2008/03/13)

10. Nakajima T, Kinoshita T, Nashimoto A, Sairenji M, Yamaguchi T, Sakamoto J, et al. Randomized controlled trial of adjuvant uracil-tegafur versus surgery alone for serosa-negative, locally advanced gastric cancer. Br J Surg. 2007;94(12):1468-76. https ://doi.org/10.1002/bjs.5996 (Epub 2007/10/20)

11. Smalley SR, Benedetti JK, Haller DG, Hundahl SA, Estes NC, Ajani JA, et al. Updated analysis of SWOG-directed intergroup study 0116: a phase III trial of adjuvant radiochemotherapy versus observation after curative gastric cancer resection. J Clin Oncol. 2012;30(19):2327-33. https://doi.org/10.1200/jco.2011.36.7136 (Epub 2012/05/16)

12. Park SH, Sohn TS, Lee J, Lim DH, Hong ME, Kim KM, et al. Phase III trial to compare adjuvant chemotherapy with capecitabine and cisplatin versus concurrent chemoradiotherapy in gastric cancer: final report of the adjuvant chemoradiotherapy in stomach tumors trial, including survival and subset analyses. J Clin Oncol. 2015;33(28):3130-6. https://doi.org/10.1200/ jco.2014.58.3930 (Epub 2015/01/07)

13. Ajani JA, Damico TA, Almhanna K, Bentrem DJ, Chao J, Das P, et al. Gastric Cancer, Version 3.2016; Clinical Practice Guidelines in Oncology. J Natl Comprehensive Cancer Netw. 2016;14(10):1286-312.

14. Japanese gastric cancer treatment guidelines 2014 (ver. 4] Gastric Cancer. 2016;20(1):1-19. https://doi.org/10.1007/s1012 0-016-0622-4

15. Fuse $\mathrm{N}$, Bando $\mathrm{H}$, Chin $\mathrm{K}$, Ito $\mathrm{S}$, Yoshikawa $\mathrm{T}$, Tsuburaya A, et al Adjuvant capecitabine plus oxaliplatin after D2 gastrectomy in Japanese patients with gastric cancer: a phase II study. Gastric Cancer. 2017;20(2):332-40.

16. Shitara K, Chin K, Yoshikawa T, Katai H, Terashima M, Ito S, et al. Phase II study of adjuvant chemotherapy of S-1 plus oxaliplatin for patients with stage III gastric cancer after D2 gastrectomy. Gastric Cancer. 2017;20(1):175-81. https://doi.org/10.1007/s1012 0-015-0581-1 (Epub 2015/12/03)

17. Sasako M, Inoue $\mathrm{M}$, Lin J, Khor C, Yang H, Ohtsu A. Gastric cancer working group report. Jpn J Clin Oncol. 2010;40.

18. Lumley T. Network meta-analysis for indirect treatment comparisons. Stat Med. 2002;21(16):2313-24.

19. Rucker G. Network meta-analysis, electrical networks and graph theory. Res Synthesis Methods. 2012;3(4):312-24.

20. Song F, Altman DG, Glenny A, Deeks JJ. Validity of indirect comparison for estimating efficacy of competing interventions: empirical evidence from published meta-analyses. BMJ. 2003;326(7387):472

21. Dias S, Sutton AJ, Ades AE, Welton NJ. Evidence synthesis for decision making 2: a generalized linear modeling framework for pairwise and network meta-analysis of randomized controlled trials. Med Decis Making. 2013;33(5):607-17. https://doi. org/10.1177/0272989X12458724. (Epub 2012/10/30)

22. Gs HJP. Cochrane handbook for systematic reviews of interventions version 5.1.0 (updated March 2011]. Naunyn-Schmiedebergs 
Archiv für experimentelle Pathologie Pharmakologie. 2014;5(2):S38.

23. Greene FL. AJCC Cancer Staging Manual (6th Edition).

24. Higgins JPT, Altman DG, Gøtzsche PC, Jüni P, Moher D, Oxman $\mathrm{AD}$, et al. The Cochrane Collaboration's tool for assessing risk of bias in randomised trials. Bmj. 2011;343(oct18 2):d5928-d.

25. Liberati A, Altman DG, Tetzlaff J, Mulrow C, Gotzsche PC, Ioannidis JP, et al. The PRISMA statement for reporting systematic reviews and meta-analyses of studies that evaluate health care interventions: explanation and elaboration. J Clin Epidemiol. 2009;62(10]):e1-34. https://doi.org/10.1016/j.jclin epi.2009.06.006

26. Tierney JF, Stewart LA, Ghersi D, Burdett S, Sydes MR. Practical methods for incorporating summary time-toevent data into meta-analysis. Trials. 2007;8:16. https://doi. org/10.1186/1745-6215-8-16

27. Dias S, Welton NJ, Caldwell DM, Ades AE. Checking consistency in mixed treatment comparison meta-analysis. Stat Med. 2010;29:932-44.

28. Salanti G, Ades AE, Ioannidis JP. Graphical methods and numerical summaries for presenting results from multiple-treatment meta-analysis: an overview and tutorial. J Clin Epidemiol. 2011;64(2):163-71. https://doi.org/10.1016/j.jclinepi.2010.03.016 (Epub 2010/08/07)

29. Tsuburaya A, Yoshida K, Kobayashi M, Yoshino S, Takahashi M, Takiguchi N, et al. Sequential paclitaxel followed by tegafur and uracil (UFT] or S-1 versus UFT or S-1 monotherapy as adjuvant chemotherapy for $\mathrm{T} 4 \mathrm{a} / \mathrm{b}$ gastric cancer (SAMIT]: a phase 3 factorial randomised controlled trial. Lancet Oncol. 2014;15(8):88693. https://doi.org/10.1016/s1470-2045(14)70025-7 (Epub 2014/06/24)

30. Cascinu S, Labianca R, Barone C, Santoro A, Carnaghi C, Cassano A, et al. Adjuvant treatment of high-risk, radically resected gastric cancer patients with 5-fluorouracil, leucovorin, cisplatin, and epidoxorubicin in a randomized controlled trial. J Natl Cancer Inst. 2007;99(8):601-7. https://doi.org/10.1093/jnci/djk131 (Epub 2007/04/19)

31. Lee JJ, Kim SY, Shin IS, Cho KS, Joo HZ, Yoon C, et al. Randomized phase III trial of cisplatin, epirubicin, leucovorin, 5-fluorouracil (PELF] combination versus 5-fluorouracil alone as adjuvant chemotherapy in curative resected stage III gastric cancer. Cancer Res Treatment. 2004;36(2):140-5. https://doi.org/10.4143/ crt.2004.36.2.140 (Epub 2004/04/01)

32. Zhu WG, Xua DF, Pu J, Zong CD, Li T, Tao GZ, et al. A randomized, controlled, multicenter study comparing intensity-modulated radiotherapy plus concurrent chemotherapy with chemotherapy alone in gastric cancer patients with D2 resection. Radiother Oncol J Eur Soc Therapeutic Radiol Oncol. 2012;104(3):361-6. https://doi.org/10.1016/j.radonc.2012.08.024 (Epub 2012/09/19)
33. Nakajima T, Nashimoto A, Kitamura M, Kito T, Iwanaga T, Okabayashi K, et al. Adjuvant mitomycin and fluorouracil followed by oral uracil plus tegafur in serosa-negative gastric cancer: a randomised trial. Lancet (London England). 1999;354(9175):273-7. https://doi.org/10.1016/S0140-6736\%2899\%2901048-X

34. Kwon HC, Kim MC, Kim KH, Jang JS, Oh SY, Kim SH, et al. Adjuvant chemoradiation versus chemotherapy in completely resected advanced gastric cancer with D2 nodal dissection. Asia Pac J Clin Oncol. 2010;6(4):278-85. https://doi.org/10.111 1/j.1743-7563.2010.01331.x (Epub 2010/12/01)

35. Chaimani A, Higgins JPT, Mavridis D, Spyridonos P, Salanti G. Graphical tools for network meta-analysis in STATA. Plos One. 2013;8(10).

36. Sterne JA, Sutton AJ, Ioannidis JP, Terrin N, Jones DR, Lau J, et al. Recommendations for examining and interpreting funnel plot asymmetry in meta-analyses of randomised controlled trials. BMJ. 2011;343:d4002. https://doi.org/10.1136/bmj.d4002 (Epub 2011/07/26)

37. Shen L, Shan Y-S, Hu H-M, Price TJ, Sirohi B, Yeh K-H, et al. Management of gastric cancer in Asia: resource-stratified guidelines. Lancet Oncol. 2013;14(12):e535-e47. https://doi. org/10.1016/s1470-2045(13)70436-4.

38. Takahari D, Hamaguchi T, Yoshimura K, Katai H, Ito S, Fuse $\mathrm{N}$, et al. Survival analysis of adjuvant chemotherapy with S-1 plus cisplatin for stage III gastric cancer. Gastric Cancer. 2014;17(2):383-6. https://doi.org/10.1007/s10120-013-0264-8 (Epub 2013/05/31)

39. Fujitani K, Tamura S, Kimura Y, Tsuji T, Matsuyama J, Iijima S, et al. Three-year outcomes of a phase II study of adjuvant chemotherapy with S-1 plus docetaxel for stage III gastric cancer after curative D2 gastrectomy. Gastric Cancer. 2014;17(2):348-53. https://doi.org/10.1007/s10120-013-0273-7 (Epub 2013/06/06)

40. Nakamura Y, Yamanaka T, Chin K, Cho H, Katai H, Terashima M, et al. Three-year outcomes of two phase II studies of adjuvant chemotherapy with S-1 plus oxaliplatin or capecitabine plus oxaliplatin in patients with stage III gastric cancer after D2 gastrectomy. American Society of Clinical Oncology; 2018.

41. Edge SB, Compton CC. The American Joint Committee on Cancer: the 7th Edition of the AJCC Cancer Staging Manual and the Future of TNM. Ann Surg Oncol. 2010;17(6):1471-4.

42. Bamias A, Karina M, Papakostas P, Kostopoulos I, Bobos M, Vourli G, et al. A randomized phase III study of adjuvant platinum/docetaxel chemotherapy with or without radiation therapy in patients with gastric cancer. Cancer Chemother Pharmacol. 2010;65(6):1009-21. https://doi.org/10.1007/s00280-010-12566 (Epub 2010/02/05) 